\title{
Radiological emergency response for community agencies with cognitive task analysis, risk analysis, and decision support framework
}

\author{
Travis S. Meyer ${ }^{\mathrm{a},}$, Joseph Z. Muething ${ }^{\mathrm{a}}$, Gustavo Amoras Souza Lima ${ }^{\mathrm{b}}$, Breno Raemy Rangel Torres ${ }^{\mathrm{b}}$, \\ Trystyn Keia del Rosario ${ }^{\mathrm{a}}$, José Orlando Gomes ${ }^{\mathrm{c}}$, and James H. Lambert ${ }^{\mathrm{d} *}$ \\ ${ }^{a}$ Univ. of Virginia, Charlottesville, VA, USA \\ ${ }^{b}$ Univ. Federal do Rio de Janeiro, Rio de Janeiro, Brazil \\ ${ }^{\mathrm{c}}$ Graduate Program in Informatics \& Industrial Engineering Department, Univ. Federal do Rio de Janeiro \\ Rua Afonso Pena, 187/403, Rio de Janeiro, Brazil, 20170-144 \\ joseorlando@nce.ufrj.br \\ ${ }^{d}$ Department of Systems and Information Engineering, University of Virginia, Charlottesville, VA
}

\begin{abstract}
Radiological nuclear emergency responders must be able to coordinate evacuation and relief efforts following the release of radioactive material into populated areas. In order to respond quickly and effectively to a nuclear emergency, highlevel coordination is needed between a number of large, independent organizations, including police, military, hazmat, and transportation authorities. Given the complexity, scale, time-pressure, and potential negative consequences inherent in radiological emergency responses, tracking and communicating information that will assist decision makers during a crisis is crucial. The emergency response team at the Angra dos Reis nuclear power facility, located outside of Rio de Janeiro, Brazil, presently conducts emergency response simulations once every two years to prepare organizational leaders for real-life emergency situations. However, current exercises are conducted without the aid of electronic or software tools, resulting in possible cognitive overload and delays in decision-making. This paper describes the development of a decision support system employing systems methodologies, including cognitive task analysis and human-machine interface design. The decision support system can aid the coordination team by automating cognitive functions and improving information sharing. A prototype of the design will be evaluated by plant officials in Brazil and incorporated to a future trial run of a response simulation.
\end{abstract}

Keywords: Preparedness; disaster; human factors; organizational behavior; risk management; cognitive engineering

\section{Introduction}

The need to ensure a rapid and effective response to a nuclear emergency has taken an especially serious tone following the recent earthquake and tsunami in Japan and the consequential critical failures in several reactors at the Fukushima Daiichi nuclear power plant [1]. In the weeks following the initial disaster, several criticisms have arisen from international nuclear regulatory agencies, which claim that the re- sponse team in Japan used questionable methods in containing the nuclear fallout and that the release of information about the incident to governmental agencies and the public was sparse and contradictory [2]. These concerns have caused several other governments and nuclear agencies to closely reevaluate their own nuclear emergency response plans. Existing research stresses the challenges posed by radiological hazards [3] [4] [5] [6] [7]. Given the rising public doubt over the safety of nuclear power brought about

\footnotetext{
* Corresponding author. Email: lambert@virginia.edu.
} 
by the events in Japan, governments are under pressure to ensure that they will be able to protect their citizens in the case of a nuclear accident.

In March 2011, the Brazilian government created the monitoring agency to review and analyze the ongoing response efforts in Japan. From this study, the agency will analyze and compare the response to Brazil's own nuclear emergency response protocols. Government officials have said that based on the results of the analysis, experts might recommend modifications to safety plans and protocols at Brazilian nuclear power plants [8]. With this new investigation, a systems-oriented study of Brazil's nuclear emergency response planning comes at an opportune time.

\section{Background}

Figure 1 shows the Angra dos Reis nuclear power facility. The Angra dos Reis facility, which is located outside of Rio de Janeiro, Brazil, relies on its response team to coordinate the evacuation and sheltering of the local community in the event of a nuclear emergency. These responders work in teams that consist of members from several organizations, all of whom must be able to cooperate, exchange resources, rapidly analyze large amounts of information, and determine optimal decisions. The team conducts emergency response simulations once every two years in partnership with the nuclear reactor facility in order to prepare organizational leaders for a reallife emergency situation. The simulations currently consist of the movement of personnel vehicles in the

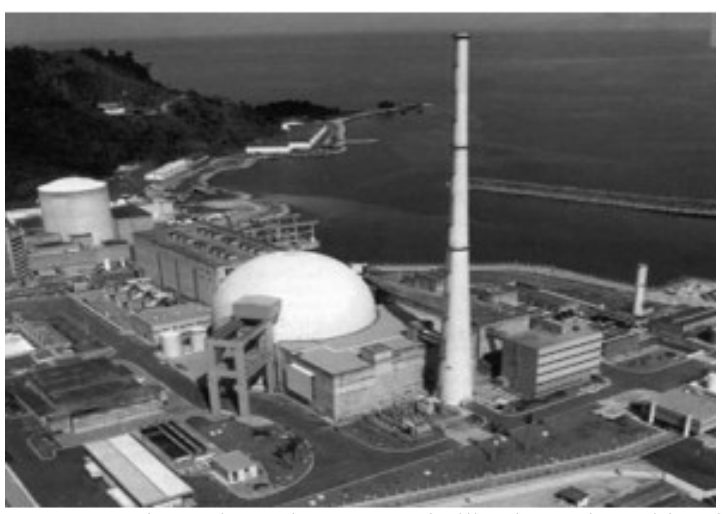

Fig. 1. Angra dos Reis nuclear power facility located outside of Rio de Janeiro, Brazil. The facility is Brazil's only operational nuclear power plant and uses emergency simulation exercises to prepare its response team.

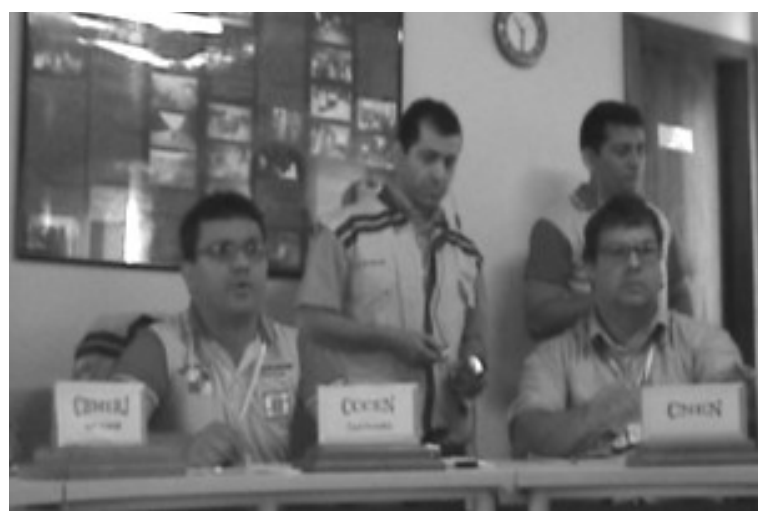

Fig. 2. A response team coordinator (left) leads the 2007 response simulations for radiological emergencies at the Angra dos Reis nuclear plant in Brazil. The current simulations are conducted with no electronic aids, which result in frequent slowdowns during the simulation and limit the plant's ability to analyze the performance of participants.

field, such as helicopters and buses, as well as evacuation protocols by military and police. The response team within the command center, which receives hypothetical scenarios and situational updates scripted by plant officials, coordinates these physical activities. While decision making within the response team is a collaborative effort, the final approval of all orders to the field lies with a designated response coordinator, a member of The Center for Control and Coordination of Nuclear Emergencies (CCCEN). Figure 2 shows the response team coordinator participating in a simulation. Examples of necessary decision making include: whether to order an evacuation or shelter, whether to distribute anti-radiation medications, and how to distribute information to the media [9].

While all organizations associated with the simulation possess their own written plans describing their responsibilities within the emergency response effort, the External Emergency Plan of Rio de Janeiro State (PEE/RJ) dictates the general protocols for responding to a nuclear accident in Angra. The plan, created by the Health and Civil Defense Ministry, contains information concerning the factors under which an unusual situation is deemed an emergency situation and a response team is ordered to convene. Figure 3 shows the classifications used in the plan for prioritizing response and relief objectives [10]. The external response plan is an important document for creating a baseline of understanding for any study and analysis of the simulation. 


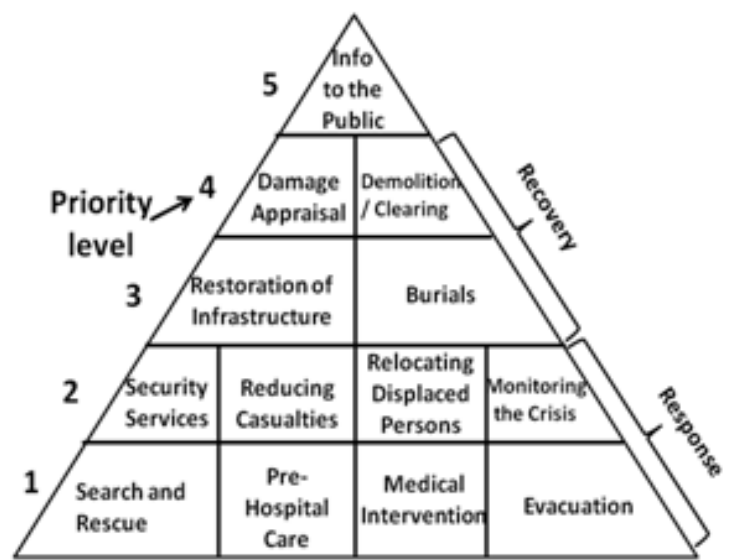

Fig. 3. Prioritization pyramid for classifying objectives for nuclear emergency response. The base level contains the most critical and immediate objectives. Upper levels contain less critical and timely objectives.

Previous studies conducted on the simulations have noted that the actions of the simulation team coordinator are of particular importance to the execution and command of the exercise. As a result, there is potential for cognitive overloading, generating a bottleneck on the decision-making process. Review of past simulation recordings suggests several deficiencies. Once the written scenarios are introduced to the response team via sealed envelope, discussion and decision making by the representatives proceed with no means of electronic data management. Individuals record their own updates with handwritten notes. Thus, if a person wants to know about the specific location or quantity of relief materials, he/she must directly ask the organization head responsible for those materials. Formal decisions are recorded only on a single spreadsheet displayed to the team on a projector in the front of the room. Studies of the simulation have revealed other weaknesses in its structure. All decisions must be approved by the coordinator, which further contributes to the information bottlenecking problem. Similarly, there is a lack of visual and communication technology support within the simulation design [11].

\section{Purpose and Scope}

Addressing the cognitive demands placed on simulation participants is a critical need. A starting point is to design a system which displays and manages data used to make decisions. Considerations for a software solution are limited to the response effort following a nuclear emergency, rather than prevention or preparation. As the present simulation deals only with emergency actions conducted outside the plant, the solution must be limited to the external response. Furthermore, any solution implemented must not change the underlying structure of the simulations currently conducted. Finally, the scope is limited to system design; implementation and testing are future work.

In assessing the quality of the solution, designs of the system require client validation. The short-term evaluation of success is conducted through internal reviews and satisfaction surveys presented to members of the client organization. The system is intended for integration into future simulations following experimental designs and revisions. The metrics used to evaluate the success of the incorporated system are decreases in the rate of mistake-related actions and the average number and duration these mistakes. These metrics are the long-term measures of success for the project, following implementation.

\section{Methods}

In keeping with systems engineering thinking, several iterative methodologies from different engineering disciplines are integrated in order to create the software solution. This holistic approach includes analysis from areas, including policy analysis, work domain analysis, cognitive engineering, and humanmachine interface design. The initial study analyzes the compliance of the plant's current emergency response plan with international nuclear safety standards. Next, work domain analysis followed by a formal cognitive task analysis is conducted on past simulation recordings. These analyses reveal critical areas in the simulation process where cognitive strain on the participants is highest. These insights are then used as a baseline in conducting an applied cognitive work analysis, a formalized methodology used to develop functional requirements for decision support systems. Finally, accepted human-machine interface design principles are employed to translate the functional requirements into a decision support system interface design to be used by the nuclear emergency responders once implemented. Aside from these combined systems methodologies, interviews and meetings with plant managers and simulation participants show the context of the actions taken and validate the ongoing work. 


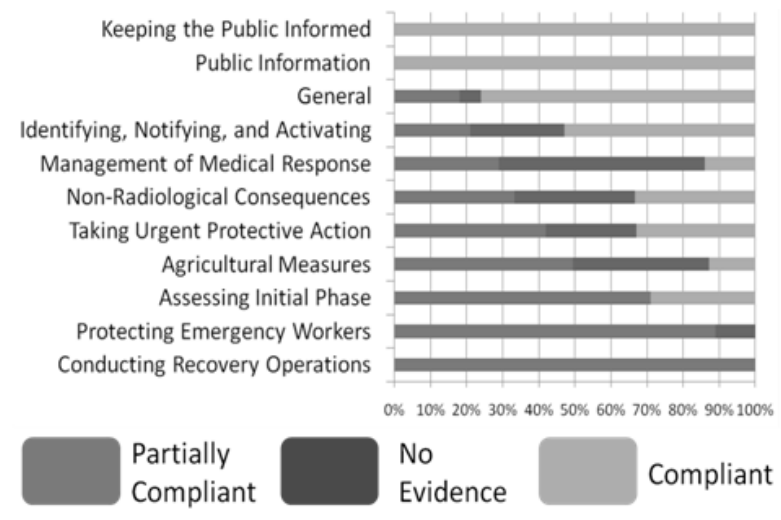

Fig. 4. Compliance assessment of the Angra dos Reis radiological response plan to 11 categories of International Atomic Energy Agency (IAEA) standards. IAEA recommendations are the global standard for radiological preparedness.

\subsection{Benchmarking of External Emergency Response Plan}

The first step is an analysis of the Angra dos Reis External Emergency Response Plan to gain familiarity with response protocol and actions. Only the parts of this 70-page document that dealt with response efforts are reviewed. To help the plan managers ensure that the current plan employed response policies are accepted by the international nuclear community, the PEE/RJ's procedures are compared against the International Atomic Energy Agency's (IAEA) own safety standards [12]. Figure 4 shows the categories for response activities rate of compliance measurements in the Brazilian response plan using the international standards. When deficiencies appear, examples of compliant policies from Virginia's Radiological Emergency Response Plan are included as a point of reference. Findings are then submitted to the plant managers for review [13].

\subsection{Analysis of Simulation Videos}

Analysis of video recordings from the 2009 simulation provides background on the simulation structure and is the next step in creating a solution. The 2007 simulation videos, which are longer and feature better sound quality than the 2009 videos, serve as the central resource in understanding what occurs during a simulated emergency. By transcribing the four hours of simulation footage into forty pages of Portuguese text, it is possible to record the dialogue and actions featured in each video. The activities from these transcripts are defined and differentiated using concepts from ergonomics, such as spatial demand, mental workload, and physical constraints [14]. Activities are coded based on individual activity type and then grouped into one of three categories: communication, order / command, and physical / technology [11].

\subsection{Cognitive Task Analysis}

Cognitive task analysis (CTA) can be defined as the study of cognition in real-world contexts and professional practices at work [15]. Use of the cognitive action criteria and coding from the transcripts opens the simulation to cognitive domain assessment. The coded activities reveal the frequency and composition of different types of activities performed throughout the simulation. Actions related to communication dominate in frequency, especially conversation in small groups (17.4\%) and explanation of details and specific knowledge (18\%). The CTA shows that the high frequency of small group discussions is accompanied by 32 specific instances of one group asking

TABLE I

ATION COGNITIVE TASK CODED ACTIVITIES IN SIMULATION
ANALYSIS

\begin{tabular}{|c|c|c|c|}
\hline Group & Activity & Count & Percentage \\
\hline \multirow{6}{*}{ Communication } & Conversation in small groups & 118 & $17.4 \%$ \\
\hline & Messages from plant & 78 & $11.5 \%$ \\
\hline & Explanation of details & 122 & $18.0 \%$ \\
\hline & External communication & 52 & $7.7 \%$ \\
\hline & Repeating known information & 32 & $4.7 \%$ \\
\hline & Question & 72 & $10.6 \%$ \\
\hline \multirow{5}{*}{$\begin{array}{c}\text { Order / } \\
\text { Command }\end{array}$} & Command to team members & 41 & $6.0 \%$ \\
\hline & $\begin{array}{l}\text { Approving and making } \\
\text { decisions }\end{array}$ & 44 & $6.5 \%$ \\
\hline & Request for silence & 21 & $3.1 \%$ \\
\hline & Request for information & 33 & $4.9 \%$ \\
\hline & Technology problems & 11 & $1.6 \%$ \\
\hline \multirow{3}{*}{$\begin{array}{l}\text { Physical / } \\
\text { Technology }\end{array}$} & People anival & 21 & $3.1 \%$ \\
\hline & Technology solutions & 9 & $1.3 \%$ \\
\hline & Confirmation Expression & 24 & $3.5 \%$ \\
\hline
\end{tabular}

Table 1 . Each activity was counted for every time it occurred in the transcript of the 2007 emergency response simulation for nuclear emergencies in Angra dos Reis, Brazil. 


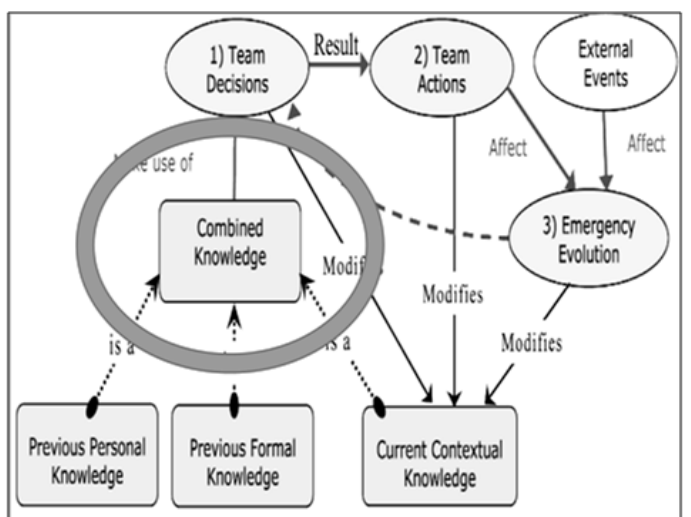

Fig.. 5. Conceptual map of knowledge during an emergency response. The decision support system will aid the radiological response team by presenting the combined forms of knowledge into an efficient display. Team decisions and actions can then be made more quickly and accurately.

either the coordinator or another group to repeat information that had already been discussed. This suggests the fragmentation in communication is causing information asymmetries. Since no electronic means are used to record prior information, information overload causes a bottleneck and slows down the simulation. In a real-world scenario, when a small delay in response can affect the lives of thousands of residents, these delays must be avoided. Table I shows the categories and activities that were coded, as well as the frequency and overall percentage of each activity.

These findings suggest the software solution should be focused on the coordinator, since all decisions and information flow through him. Moreover, the system should be able to quickly retrieve previously discussed information so as to minimize the delay in progress. Figure 5 shows the conceptual map of the knowledge required during an emergency response and where the software system should position itself within the process [16].

\subsection{Applied Cognitive Work Analysis and} Development of Functional Requirements List

Applied cognitive work analysis (ACWA) is a methodology which builds on CTA in order to bridge the gap between the initial cognitive analysis and the creation of a formal functional requirements list. ACWA consists of a sequence of small and manageable engineering transformations of conceptual models to narrow the focus of the system design [17]. Beginning with an abstract analysis of the domain and goals of the system, the steps that ultimately lead to a set of functional requirements are the creation of a Functional Abstraction Network (FAN), Cognitive Work Requirements (CWR), Information / Relationship Requirements (IRR), Representation Design Requirements (RDR) and Presentation Design Concepts $(P D C)$ [18]. Figure 6 shows an example node from the created FAN and its transformations.

\subsection{Decision Support System Design}

The end product of the ACWA is a list of functional requirements that describe the necessary aspects of a software solution. A review of the functional requirements suggests the best solution is a decision support system (DSS). A DSS is explicitly designed to solve problems with ill-defined structure where the objectives of the decision maker and the problem itself cannot be fully defined. It can support a range of decision-making styles and is easily adapted to the needs of the user as they change over time. The functional requirements stress data visualization. Geographical information systems (GIS) are designed to support capturing, storing, manipulating, analyzing and displaying spatial data [19]. Therefore, to fully satisfy the functional requirements, the DSS software solution integrates GIS design elements.

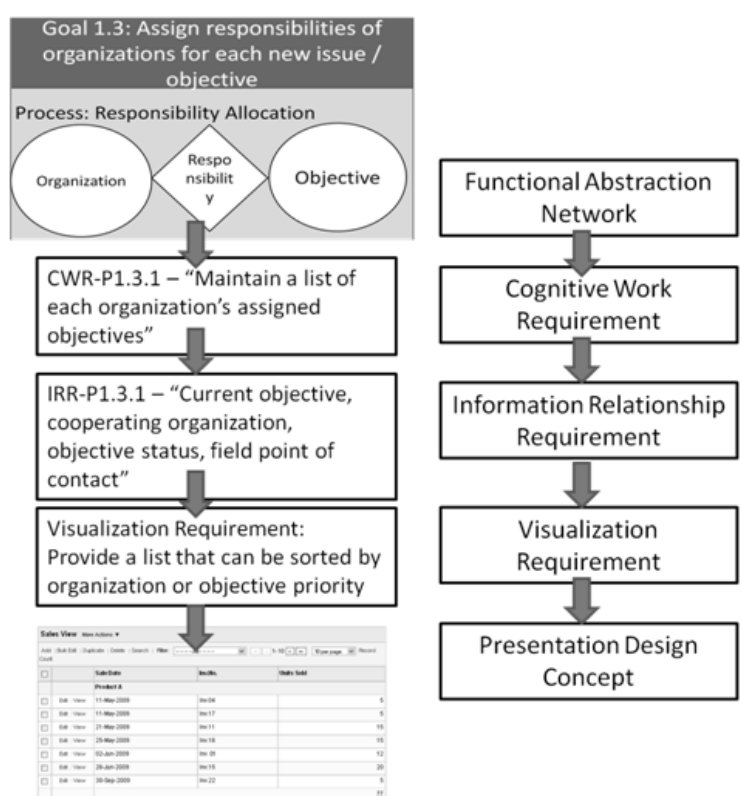

Fig. 6. Example of the applied cognitive work analysis methodology. The five transformations allow for an initial goal abstraction to be translated in to a concrete design element in an eventual decision support system for the nuclear response team. 


\section{Results and Discussion}

Figure 7 describes the DSS interface design and its panel system. The large pane shows a geographic visualization of data customized through filter selections. Examples of filter options are traffic congestion, topography, and medical sites. The side panel on the right can alternate between an objectives list, a live-feed of data updates being tracked by the system, and a search results display that permits quick access to data such as contact information.

The geographic view is constructed with multiple display layers to enable custom views such as tracking only shelter and triage locations. Providing information in this medium allows decision makers to select data and output easily and intuitively, while remaining flexible to the situational needs [19]. The location being viewed and the zoom-level can be modified by the user with the limitation that only the Angra dos Reis region has pre-populated information. The geospatial data is augmented information embedded into the data object available for display onclick. As shown in Figure 7, objects like hospitals contain information on capacity, proximity to the plant, and contact information. Weather data on wind speed and direction is displayed on the map regardless of which filters are selected as this information is vital towards any response decisions. The multipurpose side panel permits flexibility in what information to focus on. The objectives list shows competition status and prioritization. The live-feed panel tracks each information update in the system. Livefeed data can be filtered by priority level, data type, and time period. The use of icons and data type based color coding in the live-feed display increases at-aglance information content. The search feature reduces the time needed to find desired information provided it exists in the system.

All data updates track the person who provided the update and the time the update was made. In an emergency, it is critical that every piece of information has these identifiers so that decision makers know the level of accuracy of the data at the current time and can easily obtain more information by contacting the source [20]. Prioritization can be embedded into data objects as they are entered into the system with the coordinator being able to flag the criticality of new information or objectives. Coordinators have stressed the need for an overall picture of the disaster, which highlights the most pressing issues to allow for better improvisation in their response [21]. Data are to be entered by the on-site technical support

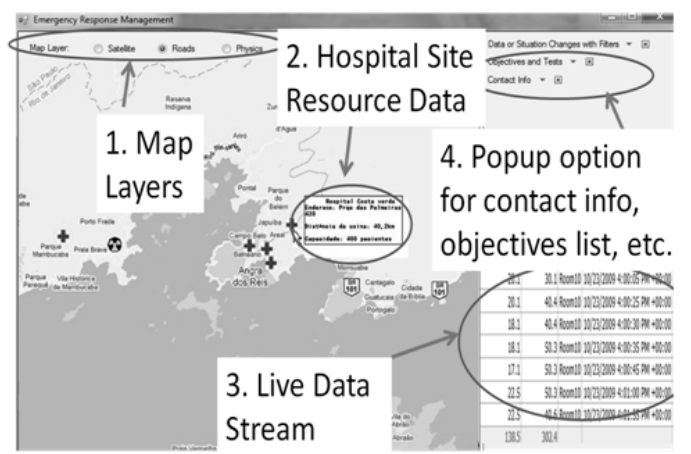

Fig. 7. Example of the decision support system interface. Example feature include: 1. Optional map layers, such as weather patterns, topography, or traffic congestion; 2. Clickable location pins (ex. Hospital), which show additional information; 3 . Live data stream of situational changes; 4. Popup windows for functions such as organizational contact info and objectives priorities list.

team through a separate interface, freeing the coordinator from managing the inflow of information from external communications and different team members. In addition to permitting the inflow of live information, the interface envisions a pre-populated database with relevant static information such as contact information, facility locations and resources, and a weather feed. The interface designs are coded in the Visual Basic programming language, allowing for quick modifications and easing the future transition process from design to implementation.

Evaluation of the solution has thus far been limited to client approval of the functional requirements. Qualitative assessment of the interface design is pending as of this report's submission. The efficacy of the DSS solution is to be measured during its implementation in the next simulation. A successful design would be shown to reduce the repetition of known information and clarifying details, while increasing the decision making contributions of the coordinator.

Incidences of technical problems should be less frequent than the current system. These assessments can be measured after future simulations by repeating the CTA procedure described in this report.

\section{Future Work}

The design is the starting point for implementation. The next efforts must focus on the architecture on which the interface can be integrated, so that the front-end has the necessary support structure. This is especially relevant when considering a number of the proposed features in the interface design, such as 
dynamic vehicle tracking, which would require infrastructure developments to ensure GPS and network technology in the real-world matches the system design. Sustained dialogue with the response coordinator is needed to ensure the tools are applicable and user-friendly. Furthermore, measuring the impact of the DSS on simulation activity will be critical in assessing and refining the efficacy of the system. Future work should include insights from the investigation by Brazilian experts of the Fukushima response. Continued developments will translate into a system that improves emergency response both during simulations and in the event of an actual emergency.

\section{Conclusions}

Responding to a nuclear emergency is a task that demands access to and understanding of a substantial amount of complex and dynamic information. As demonstrated by the recent tragedy in Japan, there is a great need for a well-prepared and systemic approach towards nuclear emergency response to minimize the scale of catastrophes. For response coordinators, there is substantial potential value in using technology to aid the decision-making process. The DSS solution for the Angra dos Reis emergency response coordinator is designed to absorb cognitive demands which would otherwise slow and reduce the effectiveness of the life or death decisions made by the emergency response team. Created through the integration of established systems engineering methodologies into an iterative design approach, the proposed interface design is directly linked to requirements which have been revised and approved by members of the simulation team. The DSS interface design is a first step in implementing a technology system that addresses the challenges of response coordination. The results, based on two years of research, provide a concrete basis for leveraging the power of technology and systemic problem solving to the high-stakes world of nuclear emergency response coordination.

\section{Acknowledgement}

The authors are grateful for the leadership and contributions of the following individuals: Colonel Roberto Jorge Lucente and Major Marcus Vinicius of the Coordenador de Operações da Defesa Civil municipal da Cidade do Rio de Janeiro; Ana Carolina
Assaife, Fernanda Araujo, and Fernanda Bruno of the Universidade Federal do Rio de Janeiro; Professor Stephanie Guerlain of the University of Virginia; Art Warren of the Virginia Department of Emergency Management; Mark Penn and Ashley Ehrhart of the City of Alexandria Emergency Management Center; Perry Cogburn of the Virginia Department of Transportation. Additionally, this work was supported in part by the U.S. National Science Foundation, the U.S. Department of Education Fund for the Improvement of Post Secondary Education (FIPSE), and Coordenação de Pessoal de Ensino de Nível Superior (CAPES) of Brazil.

\section{References}

[1] Centre, U. N. (2011, March). Nuclear emergency response must be upgraded in light of Japanese crisis - UN atomic chief. Retrieved March 25, 2011, from http://www.un.org/apps/news/story.asp?NewsID $=37834 \& \mathrm{Cr}$ $=$ japan $\& \mathrm{Cr} 1$

[2] Johnson, T. (2011, March 17). Clouds Over Japan's Nuclear Response. Retrieved March 25, 2011, from Council on Foreign Relations: http://www.cfr.org/disasters/clouds-overjapans-nuclear-response/p24412

[3] Lambert, J. H. (2010). Population Behaviors in Dirty Bomb Attack Scenarios: A Survey of the National Capital Region. Charlottesville: University of Virginia Center for Survey Research.

[4] Lambert, J.H., and M.W. Farrington. (2006). Risk-based objectives for the allocation of chemical, biological, and radiological air emissions sensors. Risk Analysis. 26(6):1659-1674.

[5] Lambert, J.H. and M.W. Farrington. (2007). Cost-benefit functions for the allocation of security sensors for air contaminants. Reliability Engineering and System Safety. 92(7):930-946

[6] Lambert, J.H. and T. Turley. (2005). Priority setting for the distribution of localized hazard protection. Risk Analysis. 25(3):745-752.

[7] Karvetski, C.W., J.H. Lambert, and I. Linkov. (2011). Scenario and multiple criteria decision analysis for energy and environmental security of military and industrial installations. Integrated Environmental Assessment and Management. 7(2):228-236.

[8] AFP. (2011, March 18). Brazil Creates Technical Group to Monitor Nuclear Crisis in Japan. Retrieved March 25, 2011, from Diálogo: http://www.dialogoamericas.com/en_GB/articles/rmisa/features/regional_news/2011/0 3/18/feature-ex-1994

[9] Sorensen, J. H. (2004). Planning for protective action decision making: evacuate or shelter-in-place. Journal of Hazardous Materials. 109(1-3): 1-11.

[10] RJ, D. C. (2007). Plano de Emergência - Nas Instalações Da Central Nuclear Almirante Alvaro Alberto (CNAAA). Rio de Janeiro: Secretario de Estado De Saûde E Defesa Civil.

[11] Costa, W. S. (2008). Resilience and Brittleness in a Nuclear Emergency Response Simulation: Focusing on Team Coordi- 
nation Activity. Porto Alegre.

[12] IAEA (2002). Preparedness and Response for a Nuclear or Radiological Emergency. Vienna: IAEA.

[13] The Commonwealth of Virginia. (1999). The Commonwealth of Virginia emergency Operations Plan: Radiological Emergency Response Plan (COVRERP). Alexandria: Virginia Department of Emergency Services.

[14] Guérin, F. A. (2001). Compreender O Trabalho Para Transormá-lo: A Prática Da Ergonomia. São Paulo: Editora Blucher Ltda. 224 pp.

[15] Crandall, B. K. (2006). Working Minds: A Practitioner's Guide to Cognitive Task Analysis. Cambridge: The MIT Press. 332 pp.

[16] Diniz, V. B. (2008). Decision Making Support in Emergency Response. In F. a. Adam, Encyclopedia of Decision Making and Decision Support Technologies. Hershey: Information Science Reference.
[17] Elm, W. C. (2000). Applied Cognitive Work Analysis: A Pragmatic Methodology for Designing Revolutionary Cognitive Affordances. Pittsburgh: Aegis Research Corporation.

[18] Potter, S. G. (2000). Case Studies: Applied Cognitive Work Analysis in the Design of Innovative Decision Support. Pittsburgh: Aegis Research Corporation.

[19] Densham, P. J. (1991). Spatial decision support systems. Geographical Information Systems: Principles and applications, 1, 403-412.

[20] Turoff, M., \& Chumer, M., \& Yao. X. (2006). The Design of a Dynamic Emergency Response Management Information System (DERMIS). Annual Review of Network Management and Security, Volume 1, 101.

[21] Mendonca, D., Beroggi, G. E. G., \& Wallace, W. A. (2001). Decision support for improvisation during emergency response operations. International journal of emergency management, 1(1), 30-38 